\title{
Shell-Isolated Tip-Enhanced Raman and Fluorescence Spectroscopy
}

\section{Journal Article}

\section{Author(s):}

Huang, Ya\#Ping; Huang, Sheng\#Chao; Wang, Xiang\#Jie; Bodappa, Nataraju; Li, Chao-Yu; Yin, Hao; Su, Hai-Sheng; Meng, Meng; Zhang, Hua; Ren, Bin; Yang, Zhi\#Lin; Zenobi, Renato (1); Tian, Zhong-Qun; Li, Jian-Feng

Publication date:

2018-06-18

\section{Permanent link:}

https://doi.org/10.3929/ethz-b-000271697

\section{Rights / license:}

In Copyright - Non-Commercial Use Permitted

\section{Originally published in:}

Angewandte Chemie. International Edition 57(S 25), https://doi.org/10.1002/anie.201802892 


\title{
Shell-Isolated Tip-Enhanced Raman and Fluorescence Spectroscopy
}

\author{
Ya-Ping Huang ${ }^{+}$, Sheng-Chao Huang ${ }^{+}$, Xiang-Jie Wan Nataraju Bodappa, Chao-Yu Li, Hao Yin, Hai- \\ Sheng Su, Meng Meng, Hua Zhang, Bin Ren, ${ }^{*}$ Zhi-Lin Yang, Renato Zenobi, ${ }^{*}$ Zhong-Qun Tian, and \\ Jian-Feng Li*
}

Y. P. Huang, ${ }_{{ }^{[+]}}$S. C. Huang, ${ }_{\left.{ }^{+}\right]}$Dr. N. Bodappa, Dr. C. Y. Li, H. Yin, H. S. Su, M. Meng, Dr. H Zhang, Prof. B. Ren, Prof. R. Zenobi, Prof. Z. Q. Tian, Prof. J. F. Li, MOE Key Laboratory of Spectrochemical Analysis and Instrumentation, State Key Laboratory of Physical Chemistry of Solid Surfaces, iChEM, College of Chemistry and Chemical Engineering, Xiamen University, Xiamen 361005, China. E-mail: Li@xmu.edu.cn, bren@xmu.edu.cn

X. J. Wang, Prof. Z. L. Yang, Prof. J. F. Li, Department of Physics, Research Institute for Biomimetics and Soft Matter, Xiamen University, Xiamen 361005, China

H. Yin, Prof. R. Zenobi, Department of Chemistry and Applied Biosciences, ETH Zürich, CH-8093 Zürich, Switzerland, E-mail: zenobi@org.chem.ethz.ch

[+] These authors contributed equally to this work

\begin{abstract}
Tip-enhanced Raman spectroscopy can provide molecular fingerprint information with ultrahigh spatial resolution, but the tip will be easily contaminated, thus leading to artifacts. It also remains a great challenge to establish tip-enhanced fluorescence due to quenching resulting from the proximity of the metal tip. Herein, we report shell-isolated tip-enhanced Raman and fluorescence spectroscopies by employing ultrathin shell-isolated tips fabricated by atomic layer deposition. Such shell-isolated tips not only show outstanding electromagnetic field enhancement in TERS, but also exclude interference by contaminants, thus greatly promoting applications in solution. Tip-enhanced fluorescence has also been achieved using these shell-isolated tips, with enhancement factors up to $1.7 \times 10^{3}$, consistent with theoretical simulations. Furthermore, tip-enhanced Raman and fluorescence signals are acquired simultaneously, and their relative intensities can be manipulated by changing the shell thickness. This work opens up a new avenue for ultrahigh resolution surface analysis using plasmonenhanced spectroscopies.
\end{abstract}

Tip-enhanced Raman spectroscopy (TERS) combines the advantages of scanning probe microscopy and Raman spectroscopy. It provides both morphologic and chemical fingerprint information about the surface with a high detection sensitivity down to a singlemolecule level and a high spatial resolution down to sub-nanometers due to the lightning-rod effect and localized surface plasmon resonance (LSPR) at the tip apex. ${ }^{[1]}$ Nanostructured $\mathrm{Au}$ and $\mathrm{Ag}$, which are known to exhibit outstanding plasmonic properties in the visible, have usually been used to prepare TERS tips. A highly confined electromagnetic (EM) field created in such a nano-gap between the plasmonic tip and substrate can greatly enhance the Raman scattering of molecules nearby. ${ }^{[2]}$ However, the tip is easily contaminated by impurities from the environment, especially when working in solution. In addition, analyte molecules from the substrate may also adsorb on the tip. Both these factors would significantly interfere with the Raman information of the analytes of interest. Although the ultrahigh vacuum TERS developed recently can avoid tip contamination, it cannot be applied in systems under ambient atmosphere or solution conditions, and it requires very expensive apparatus. ${ }^{[3]}$ Another challenge of traditional TERS is that the Ag tip loses its plasmonic activity rapidly in atmospheric conditions due to the tarnishing of $\mathrm{Ag}^{\left[{ }^{[4]}\right.}$

To solve the above-mentioned problems, several strategies have been proposed. The easiest way to protect a Ag tip from oxidation is to store it in a nitrogen glovebox with low oxygen and water content, which can prolong the lifetime of the tip to several months. ${ }^{[5]}$ Storing $\mathrm{Ag}$ tips on a sacrificial metal holder that has lower reduction potential than $\mathrm{Ag}$ can also prolong its lifetime to some extent. ${ }^{[6]}$ The tip can also be protected by encapsulation with self-assembled monolayers (SAMs) of molecules whose Raman signals do not overlap the signals of the analyte..$^{[7]}$ Nevertheless, such SAMs are not stable in harsh environments and may promote the adsorption of some specific molecules thus distorting the information of the analyte of interest. ${ }^{[8]}$ Recently, the Zenobi group and the Li group have developed a wet chemical method to prepare shell-isolated tips by coating silica shells on the surface of Au or Ag tips. ${ }^{[9]}$ The shellisolated tips exhibit longer lifetime, however, this chemical method is not general and tedious to scale up, greatly limiting the application of the shell-isolated tips. Therefore, development of more general methods would be of great significance.

Atomic layer deposition (ALD) is a self-limiting thin film deposition technique without any specific requirement in terms of material and morphology of the substrate, and the deposition thickness can be precisely controlled at the subnanometer scale or even atomic level by the deposition cycles. ${ }^{[10]}$ It has been demonstrated that aluminum shells, which turn into aluminum oxide layers under air, can be coated on Ag tips via a similar physical vapor deposition method. These oxide layers improve the wear resistance and lifetime of the tip. ${ }^{[11]}$ Such tips have also been used to study catalytic reactions with high spatial resolution. ${ }^{[12]}$

Herein, we developed a general ALD method to prepare shell-isolated Au or Ag tips for tip-enhanced Raman or fluorescence spectroscopy. The shell-isolated Au or Ag tips with various types of shells including silica, aluminum oxide, and titanium oxide are successfully prepared via this method, and the shell thickness was precisely controlled in the range of 1-20 nm. Such tips exhibit strong electromagnetic enhancement and show great advantages for TERS in solution. Only the Raman signal of the analyte is enhanced while the interference from other molecules in the solution is completely excluded. Furthermore, tip-enhanced fluorescence has also been achieved by using the shell-isolated tips. The shell-isolated tips weaken the nonradiative energy transfer in scanning tunneling microscope (STM)-based tip-enhanced fluorescence, leading to a much higher fluorescence enhancement factor $\left(1.7 \times 10^{3}\right)$ compared 
to a bare tip. More importantly, Raman and fluorescence signals for a molecule can be obtained simultaneously, and the ratio between them can be easily tuned by varying the shell thickness.
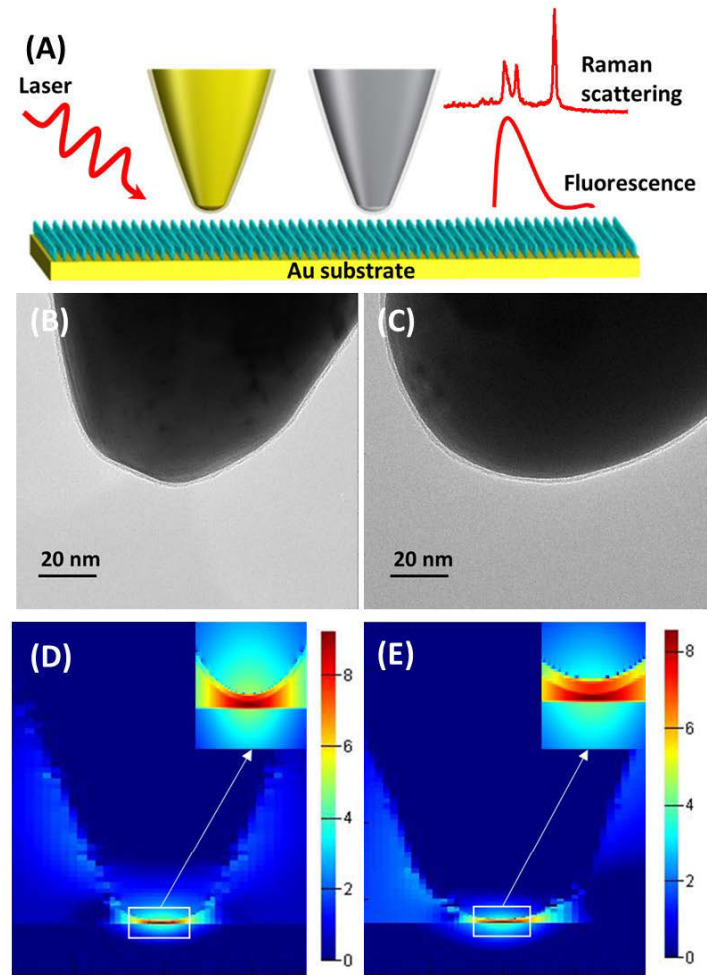

Figure 1. (A) Schematic diagram of shell-isolated plasmonic tip-enhanced spectroscopies. (B), (C) TEM images of the apex of a Au or Ag tip coated with $2 \mathrm{~nm}$ SiO, respectively. (D), (E) The three dimensional-finite difference time domain (3D-FDTD) simulation of the Raman enhancement factor in the nano-gap between the Au or Ag tip coated with $2 \mathrm{~nm}$ silica and a Au substrate, respectively. The wavelength of the incident laser is $633 \mathrm{~nm}$. The radius of the Au and Ag tip is $30 \mathrm{~nm}$ and 60 $\mathrm{nm}$, respectively. The distance between the tip and the substrate is $1 \mathrm{~nm}$.

In this work, shell-isolated $\mathrm{Au}$ and $\mathrm{Ag}$ tips were used to enhance both Raman and fluorescence signals (Figure $1 \mathrm{~A})$. Au and Ag tips were first prepared by an electrochemical etching method, then shells were coated on them via ALD (Figure S1, see Supporting Information for details). Highly uniform shells with a thickness of about $2 \mathrm{~nm}$ were coated onto Au and Ag tips as revealed by TEM images (Figures 1B and 1C). The shell thickness was flexibly tailored in the range of 1-20 nm by adjusting the number of ALD cycles (Figures S2-S3). These results indicate that the ALD method is much more general compared with the wet chemical coating method, which was difficult to use on Ag tips and often left pinholes in the coating, ${ }^{[13]}$ and can be used for coating of different types of shells onto different tips. At the same time, the shell thickness can be precisely controlled at the sub-nanoscale and shells made of other materials like nitride, sulfide, and fluoride etc. can be prepared by varying the precursors. Moreover, the preparation of such tips in large batches is possible.

To investigate the electromagnetic enhancement properties of the tips after coating them with $2 \mathrm{~nm}$ silica, three dimensional-finite difference time domain (3D-FDTD) simulations were used to calculate the Raman enhancement factors (EF) in the nano-gap between the tip and the Au substrate. As shown in Figures 1D and 1E, the EFs for $2 \mathrm{~nm}$ silica coated Au or Ag tips can still reach $10^{8}$, though 1-2 magnitudes less than those of the corresponding bare tips (Figure S4). Moreover, the same tip before and after being coated with a $2 \mathrm{~nm}$ silica shell was used to acquire the TERS signal of (4'-(pyridin-4-yl)biphenyl-4-yl)methanethiol (4-PBT), a frequently-used model molecule in TERS due to its strong Raman scattering, ${ }^{[14]}$ assembled on Au(111). The Raman signal of 4-PBT from the coated tip only slightly decreased compared to that of the bare tip (Figure S5), indicating that the tip coated with $2 \mathrm{~nm}$ silica still has strong electromagnetic enhancement performance. Further increasing the shell thickness would significantly decrease the enhancement. ${ }^{[9 a, 15]}$ Therefore, compromising between the enhancement and isolation effect, tips with $2 \mathrm{~nm}$ silica shells were used in the subsequent experiments.

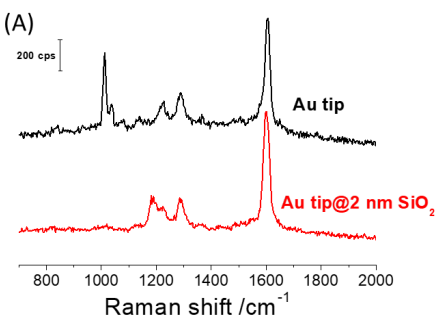

(B)

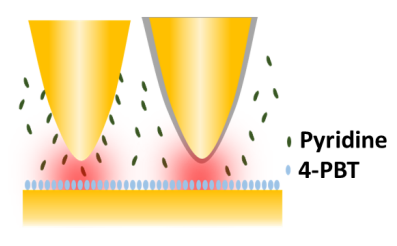

Figure 2. (A) TERS spectra of 4-PBT assembled on $\mathrm{Au}(111)$ acquired by a bare Au tip (black line) and a Au tip@2 nm SiO 2 (red line) in an aqueous solution containing $5 \mathrm{mM}$ pyridine and $10 \mathrm{mM} \mathrm{NaClO}$. The laser wavelength was $633 \mathrm{~nm}$ with $0.25 \mathrm{~mW}$ power. The power density was about $0.08 \mathrm{~mW} / \mu \mathrm{m}^{2}$, which would not cause damage to the samples. ${ }^{[16]}$ The acquisition time was $1 \mathrm{~s}$. The tunneling current was $200 \mathrm{pA}$, and the bias voltage was $600 \mathrm{mV}$. (B) Schematic diagram of TERS in a solution using a bare or shell-isolated tip. 
Expanding TERS to the solution environment is of significant importance, especially for biological systems. However, this is still a great challenge due to the interference from impurities in the solution. To demonstrate the advantage of the shell-isolated tips developed in this work, they were further employed for TERS measurements in solution. The TERS tests were carried out using the bare or $2 \mathrm{~nm}$ silica shell-isolated Au tips in a solution containing pyridine to detect 4-PBT assembled on $\mathrm{Au}(111)$ (see the experimental section and Figure S6 in Supporting Information for details). The TERS spectrum obtained by the bare Au tip (without $2 \mathrm{~nm}$ silica shell) exhibits the Raman signals of both 4-PBT and pyridine, with bands at 1185, 1286, $1603 \mathrm{~cm}^{-1}$ and 1009, $1034 \mathrm{~cm}^{-1}$, respectively (Figure $2 \mathrm{~A}$, black curve). ${ }^{[17]}$ This indicates that pyridine or other impurities in the solution can easily absorb on the bare tip, thus leading to spurious Raman signals from other species besides the analyte on the substrate (Figure 2B). In contrast, only the Raman signals of 4-PBT are observed when the Au tip coated with $2 \mathrm{~nm}$ silica shell was used (Figure 2A, red curve). These results demonstrate that the ultrathin silica shell can effectively exclude interferences from impurities by suppressing their interaction with the tip and thus help to acquire clean information of the analyte of interest. Hence, such shell-isolated tips can be applied to the TERS studies in solutions containing complex components, for example, live cells, without the limitation of impurities adsorbing on the tip.

In similar to TERS studies, tip-enhanced fluorescence has also been investigated theoretically and experimentally. ${ }^{[18]}$ In plasmonenhanced fluorescence spectroscopy, the enhanced local EM field, which is generated by the plasmonic metallic nanostructure, can accelerate the excitation rate and spontaneous emission rate of fluorophores nearby. However, the fluorophores are usually located in the nano-gap between the metal tip and metal substrate in STM-based tip-enhanced fluorescence spectroscopy, thus nonradiative energy transfer to the metal tip and metal substrate efficiently quenches the fluorescence signals. ${ }^{[19]}$ In this work, Cy5 fluorescent molecules, whose fluorescence spectra are shown in Figure S7, were immobilized on a Au(111) substrate via a coupling agent (Figures $3 \mathrm{~A}$ and S8). Figure 3B shows the tip-enhanced fluorescence spectra obtained by a Ag tip coated with $2 \mathrm{~nm}$ silica shell. The fluorescence intensity of Cy5 with the tip retracted is very low (black curve), due to efficient nonradiative energy transfer to the metal substrate. However, the spectrum of Cy5 with the tip engaged displays much higher intensity (red curve). The calculated fluorescence enhancement factor is up to about $1.7 \times 10^{3}$. Such an enhancement results from the strong localized electric field in the nano-gap between the tip and substrate, which boosts excitation and spontaneous emission processes of Cy5. The effect of the distance between Cy5 and Au substrate to the fluorescence intensity was also studied (Figure S8). The results show that increasing the distance between the fluorophore and metal substrate reduces nonradiative energy transfer, thus producing higher fluorescence signals. Furthermore, one of the most significant advantages of the silica coated Ag tip in tip-enhanced fluorescence compared to the bare tip is that it can block the nonradiative energy transfer between Cy5 and the tip, thus leading to much stronger fluorescence signals (Figure S9).

To understand the fundamental processes of the shell-isolated tip-enhanced fluorescence, 3D-FDTD calculations were used to simulate the effect of the EM field on the excitation and emission efficiency as well as the nonradiative decay rate. ${ }^{[20]}$ The radiative decay rate enhancement, nonradiative decay rate enhancement, and quantum yield of Cy5 are plotted as a function of the wavelength of excitation light (Figure 3C). The quantum yield increases with the increase of the wavelength, and it is about 0.55 under the excitation laser used here $(633 \mathrm{~nm})$. The electromagnetic enhancement in the nano-gap between the Ag tip coated with $2 \mathrm{~nm}$ silica and the Au substrate with $633 \mathrm{~nm}$ laser irradiation is presented in Figure 3D. A highly enhanced localized EM field is observed in the nano-gap under these conditions. This enhanced localized EM field can increase the excitation and emission efficiency as well as the nonradiative decay rate to the metal substrate and metal tip. The final fluorescence enhancement factor is a balance of these processes and can be calculated according to:

$$
E F_{f l u}=|E|^{2}\left(\frac{q}{q^{0}}\right)
$$

Where $\mathrm{E}, \mathrm{q}$, and $\mathrm{q}^{0}$ are the EM field enhancement, the quantum yield with and without electromagnetic enhancement, respectively. ${ }^{[21]}$ The calculated value is $1.4 \times 10^{3}$, which agrees very well with the experimental result $\left(1.7 \times 10^{3}\right)$. Such a high enhancement factor indicates that the shell-isolated

tip-

(A)

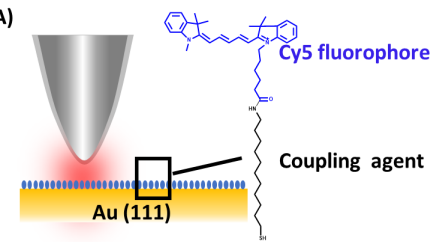

(C)

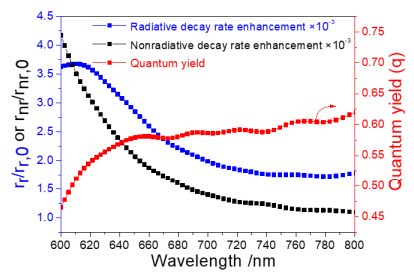

(B)

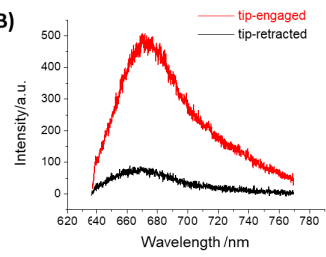

(D)

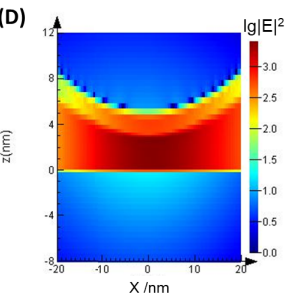

Figure 3. (A) schematic diagram of shell-isolated tip-enhanced fluorescence. The probe molecule, Cy5, was immobilized on the Au(111) surface with 11-amino-1undecanethiol hydrochloride. (B) Tip-enhanced fluorescence spectra obtained by Ag tip coated with a $2 \mathrm{~nm} \mathrm{SiO}_{2}$ shell. The black and red curves are the spectra with the tip retracted and the tip engaged, respectively. A $633 \mathrm{~nm}$ laser with a power of $25 \mu \mathrm{W}$ was used as the excitation light. The acquisition time was $1 \mathrm{~s}$. The tunneling current was $200 \mathrm{pA}$, and the bias voltage was $600 \mathrm{mV}$. (C) 3D-FDTD theoretical calculation of radiative decay rate enhancement ( $\left.\mathrm{Y}_{\mathrm{r}} / \mathrm{Y}_{\mathrm{r}, 0}\right)$, nonradiative decay rate enhancement $\left(\mathrm{Ynr}_{\mathrm{nr}} / \mathrm{Ynr}_{\mathrm{nr}, 0}\right)$, and quantum yield (q) of Cy5 as a function of wavelength. (D) 3D-FDTD calculated electromagnetic enhancement in the nanogap between the Ag@2 nm $\mathrm{SiO}_{2}$ tip and Au substrate. 
enhanced fluorescence is a promising technique to realize fluorescence imaging with ultra-high spatial resolution.

Fluorescence has very high sensitivity and affords electronic information of the analyte, whereas Raman spectroscopy can provide vibrational information. Thus, dual mode spectroscopy combining fluorescence and Raman signals possessing both of their advantages has emerged and attracted great attention recently. ${ }^{[22]}$ However, it is still very challenging to record strongly enhanced fluorescence and Raman signals simultaneously due to the completely different requirement for these two spectroscopies. Fluorescence should be acquired at a larger nano-gap to suppress quenching, whereas Raman spectroscopy needs a smaller nano-gap to generate a large local field enhancement. In this work, tip-enhanced Raman and fluorescence signals were obtained in a single spectrum using the shell-isolated tip, and their relative intensity can be easily tuned by changing the shell thickness of the tip. As shown in Figure 4 , strong Raman signals are obtained using a bare Ag tip, whereas the fluorescence is almost quenched. To overcome the nonradiative energy loss, an ultra-thin $1 \mathrm{~nm}$ silica shell is coated onto the Ag tip surface, which leads to a remarkable fluorescence enhancement as well as the characteristic Raman scattering signals of Cy5. With a further increase of the shell thickness to $2 \mathrm{~nm}$, the fluorescence signals became dominant while the vibrational Raman signals were almost disappeared.

In summary, shell-isolated tip-enhanced Raman and fluorescence spectroscopies have been investigated using shell-isolated tips in this work. Au or Ag tips coated with different shells have been successfully prepared via ALD, and the shell material as well as thickness is tuned by modulating the precursor or ALD cycle. Such shell-isolated tips exhibit strong electromagnetic enhancement capabilities and protect the tip from contamination, thus hold great potential for TERS in solutions. At the same time, the shell-isolated tip can minimize the nonradiative energy transfer caused by metallic materials in
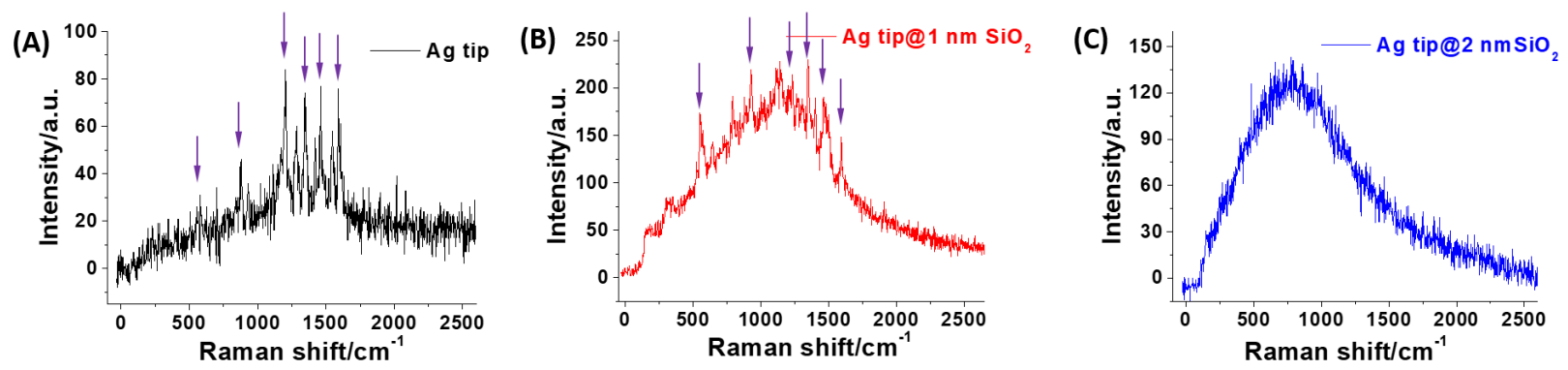

Figure 4. (A), (B), (C) Spectra obtained by bare Ag tip, Ag tip@1 nm $\mathrm{SiO}_{2}$, and Ag tip@2 nm SiO , respectively. Cy5 was functionalized on the Au film surface with 2-aminoethanethiol. The laser is $633 \mathrm{~nm}$ with $25 \mu \mathrm{W}$ power. The acquisition time is $1 \mathrm{~s}$. The tunneling current is $200 \mathrm{pA}$, and the bias voltage is $600 \mathrm{mV}$. 
tip-enhanced fluorescence, leading to a high enhancement factor of $1.7 \times 10^{3}$. More significantly, tip-enhanced Raman and fluorescence signals can be acquired simultaneously using a shell-isolated tip, and their relative intensity can be tuned by varying the shell thickness. This work paves a new avenue in plasmon-enhanced spectroscopies including fluorescence, Raman scattering, photoluminescence, etc. We also anticipate that shell-isolated tips can also be further employed in near-field enhanced mass or infrared spectroscopies with ultrahigh enhancement and spatial resolution.

\section{Acknowledgements}

This work was supported by the NSFC (21522508), the MOST (2016YFA0200601), ERC Advanced Grant (741431), the Fundamental Research Funds for the Central Universities (20720180037), and the Thousand Youth Talents Plan of China.

\section{Conflict of interest}

The authors declare no conflict of interest.

Keywords: tip-enhanced Raman spectroscopy $・$ Raman spectroscopy $\bullet$ fluorescence $\bullet$ shell-isolated tip $•$ atomic layer deposition

[1] a) R. Zhang, Y. Zhang, Z. C. Dong, S. Jiang, C. Zhang, L. G. Chen, L. Zhang, Y. Liao, J. Aizpurua, Y. Luo, J. L. Yang, J. G. Hou, Nature 2013, 498, 82-86; b) Y. Zhang, Y. Luo, Y. Zhang, Y. J. Yu, Y. M. Kuang, L. Zhang, Q. S. Meng, Y. Luo, J. L. Yang, Z. C. Dong, J. G. Hou, Nature 2016, 531, 623-627; c) J. H. Zhong, X. Jin, L. Meng, X. Wang, H.S. Su, Z. L. Yang, C. T. Williams, B. Ren, Nat. Nanotechnol. 2017, 12, 132-136; d) P. Verma, Chem. Rev. 2017, 117, 6447-6466; e) L. Xiao, Z. D. Schultz, Anal. Chem. 2018, 90, 440-458.

[2] a) E. Bailo, V. Deckert, Chem. Soc. Rev. 2008, 37, 921-930; b) J. Steidtner, B. Pettinger, Phys. Rev. Lett. 2008, 100, 236101; c) S. Berweger, J. M. Atkin, R. L. Olmon, M. B. Raschke, J. Phys. Chem. Lett. 2012, 3, 945-952.

[3] a) E. A. Pozzi, G. Goubert, N. Chiang, N. Jiang, C. T. Chapman, M. O. McAnally, A. I. Henry, T. Seideman, G. C. Schatz, M. C. Hersam, R. P. Van Duyne, Chem. Rev. 2016, 117, 4961-4982; b) Z. Zhang, L. Chen, M. Sun, P. Ruan, H. Zheng, H. Xu, Nanoscale 2013, 5, 3249-3252.

[4] M. D. McMahon, R. Lopez, H. M. Meyer, L. C. Feldman, R. F. Haglund, Appl. Phys. B 2005, 80, 915-921.

[5] N. Kumar, S. J. Spencer, D. Imbraguglio, A. M. Rossi, A. J. Wain, B. M. Weckhuysen, D. Roy, Phys. Chem. Chem. Phys. 2016, 18, $13710-13716$.

[6] L. Opilik, Ü. Dogan, J. Szczerbiński, R. Zenobi, Appl. Phys. Lett. 2015, 107, 091109.

[7] T. Schmid, B. S. Yeo, G. Leong, J. Stadler, R. Zenobi, J. Raman Spectrosc. 2009, 40, 1392-1399.

[8] a) P. Pienpinijtham, S. Vantasin, Y. Kitahama, S. Ekgasit, Y. Ozaki, J. Phys. Chem. C 2016, 120, 14663-14668; b) J. Stadler, T. Schmid, R. Zenobi, Nanoscale 2012, 4, 1856-1870.

[9] a) C. Y. Li, M. Meng, S. C. Huang, L. Li, S. R. Huang, S. Chen, L. Y. Meng, R. Panneerselvam, S. J. Zhang, B. Ren, Z. L. Yang, J. F. Li, Z. Q. Tian, J. Am. Chem. Soc. 2015, 137, 13784-13787; b) L. Opilik, Ü. Dogan, C. Y. Li, J. F. Li, R. Zenobi, J. Phys. Chem. C 2016, 120, $20828-20832$.

[10] S. M. George, Chem. Rev. 2010, 110, 111-131.

[11] C. A. Barrios, A. V. Malkovskiy, A. M. Kisliuk, A. P. Sokolov, M. D. Foster, J. Phys. Chem. C 2009, 113, 8158-8161.

[12] N. Kumar, B. Stephanidis, R. Zenobi, A. J. Wain, D. Roy, Nanoscale 2015, 7, 7133-7137.

[13] J. Lu, B. Fu, M. C. Kung, G. Xiao, J. W. Elam, H. H. Kung, P. C. Stair, Science 2012, 335, 1205-1208.

[14] Z. C. Zeng, S. C. Huang, D. Y. Wu, L. Y. Meng, M. H. Li, T. X. Huang, J. H. Zhong, X. Wang, Z. L. Yang, B. Ren, J. Am. Chem. Soc. $2015,137,11928-11931$.

[15] J. F. Li, Y. F. Huang, Y. Ding, Z. L. Yang, S. B. Li, X. S. Zhou, F. R. Fan, W. Zhang, Z. Y. Zhou, D. Y. Wu, B. Ren, Z. L. Wang, Z. Q. Tian, Nature 2010, 464, 392-395.

[16] M. Mochizuki, G. Lkhamsuren, K. Suthiwanich, E. A. Mondarte, T. Yano, M. Hara, T. Hayashi, Nanoscale 2017, 9, 10715-10720.

[17] X. Wang, J. H. Zhong, M. Zhang, Z. Liu, D. Y. Wu, B. Ren, Anal. Chem. 2016, 88, 915-921.

[18] a) J. M. Gerton, L. A. Wade, G. A. Lessard, Z. Ma, S. R. Quake, Phys. Rev. Lett. 2004, 93, 180801; b) H. G. Frey, S. Witt, K. Felderer, R. Guckenberger, Phys. Rev. Lett. 2004, 93, 200801; c) H. G. Frey, J. Paskarbeit, D. Anselmetti, Appl. Phys. Lett. 2009, 94, 241116.

[19] a) P. Anger, P. Bharadwaj, L. Novotny, Phys. Rev. Lett. 2006, 96, 113002; b) N. Chiang, N. Jiang, E. A. Pozzi, M. C. Hersam, L. Jensen, T. Seideman, R. P. Van Duyne, Nano Lett. 2015, 15, 4114-4120.

[20] L. Meng, M. Sun, J. Chen, Z. Yang, Sci. Rep. 2016, 6, 19558.

[21] A. Hartschuh, Angew. Chem. Int. Ed. 2008, 47, 8178-8191.

[22] a) F. Schleifenbaum, S. Peter, A. J. Meixner, J. Phys. Chem. A 2010, 114, 143-150; b) A. M. Gabudean, M. Focsan, S. Astilean, J. Phys. Chem. C 2012, 116, $12240-12249$. 